Travaux du Laboratoire de Rythmo-pédagogie de Paris

\title{
Marcel Jousse (1886-1961)
}

\section{Professeur d'Anthropologie linguistique} à l'École d'Anthropologie

Directeur du Laboratoire de Rythmo-pédagogie de Paris

\section{Du mimisme à la musique chez l'enfant}

\section{(1935)}

Un document produit en version numérique par M. Yves Beaupérin, bénévole, directeur de l'Institut de mimopédagogie Courriel: yves.beauperin@wanadoo.fr Site web: www.mimopedagogie.com

Dans le cadre de la collection: "Les classiques des sciences sociales"

Site web: http://www.uqac.uquebec.ca/zone30/Classiques_des_sciences_sociales/index.html

Une collection développée en collaboration avec la Bibliothèque Paul-Émile-Boulet de l'Université du Québec à Chicoutimi Site web: http://bibliotheque.uqac.uquebec.ca/index.htm 
Un document produit en version numérique par Yves Beaupérin, bénévole, directeur de l'Institut de mimopédagogie, dans le sillon de Marcel Jousse.

Courriel: yves.beauperin@wanadoo.fr

Site web: www.mimopedagogie.com

à partir de :

\section{Marcel JOUSSE (1886-1961)}

\section{Du Mimisme à la Musique chez l'enfant (1935). Mémoire.}

Une édition électronique réalisée à partir du mémoire de Marcel Jousse, Du Mimisme à la Musique chez l'enfant (1935). Mémoire publié à Paris, Librairie orientaliste Paul Geuthner, 12, rue Vavin (VIe), 1935. Travaux du Laboratoire de Rythmo-pédagogie de Paris.

Polices de caractères utilisée :

Pour le texte: Times, 12 points.

Pour les citations : Times 10 points.

Pour les notes de bas de page : Times, 10 points.

Édition électronique réalisée avec le traitement de textes Microsoft Word 2001 pour Macintosh.

Mise en page sur papier format

LETTRE (US letter), 8.5' x 11',)

Édition complétée le 6 mai 2003 à Chicoutimi, Québec.

\section{Fait avec}




\section{Table des matières}

I Le Mimisme corporel et manuel

II Le Parallélisme propositionnel

III Du Mimisme au Mimographisme

IV Le Phonomimisme auriculaire

V Le Phonomimisme oral

VI Du Mimage au Langage

VII Du Langage au Style oral

VIII Du Style oral à la Musique 


\section{REMERCIEMENTS}

L'Association Marcel Jousse, par l'intermédiaire de son président, M. Jean-Ghislain d'Eudeville, nous a généreusement accordée, le 17 avril 2003, la permission de diffuser, sur le site internet Les Classiques des sciences sociales, les œuvres de Marcel Jousse publiées de son vivant :

Études de psychologie linguistique Le style oral rythmique et mnémotechnique chez les verbomoteurs Revue Archives de philosophie Volume II, Cahier IV 1925

La pensée et le geste. 1 : le geste mimique corporel et manuel. Revue Le manuscrit autographe. 1927

Études sur la psychologie du geste. Les rabbis d'Israël. Les récitatifs rythmiques parallèles. I Genre de la maxime, Spes, Paris, 1930

Méthodologie de la psychologie du geste. Revue des cours et conférences, n¹1, 15 mai 1931, p. 201-218

Les Lois psycho-physiologiques du Style oral vivant et leur utilisation philologique. L'Ethnographie n²3, 15 avril 1931, p.1-18

Henri Brémond et la psychologie de la lecture. In memoriam Revue des cours et conférences, décembre 1933

Du mimisme à la musique chez l'enfant, Geuthner, Paris, 1935

Mimisme humain et psychologie de la lecture, Geuthner, Paris, 1935

Mimisme humain et style manuel, Geuthner, Paris, 1936

Les outils gestuels de la mémoire dans le milieu ethnique palestinien : Le Formulisme araméen des récits évangéliques. L'Ethnographie n³0, 15 décembre 1935, p. 1-20

Le mimisme humain et l'anthropologie du langage, Revue anthropologique, Juillet-Septembre 1936, p.201-215

Le bilatéralisme humain et l'anthropologie du langage, Revue anthropologique, Avril-Septembre 1940 , p. 2-30

Judahen, Judéen, Judaïste dans le milieu ethnique palestinien. L'Ethnographie n`38, 1er Janvier1er Juillet 1946, p.3-20

Père Fils et Paraclet dans le milieu ethnique palestinien. L'Ethnographie n³9, Année 1941, p. 358

Les formules targoumiques du Pater dans le milieu ethnique palestinien L'Ethnographie $\mathrm{n}^{\circ} 42$, Année 1944, p. 4-51

La manducation de la leçon dans le milieu ethnique palestinien. Geuthner, Paris, 1950.

Rythmo-mélodisme et rythmo-typographisme pour le style oral palestinien. Geuthner, Paris, 1952

igeudeville@hotmail.com

Un grand merci à M. Rémy Guérinel, de Nanterre, d'avoir rendu possible, grâce à toutes ses démarches, ce projet de diffusion libre de l'œuvre de Marcel Jousse : (guerinel@hotmail.com ) 
Travaux du Laboratoire de Rythmo-pédagogie de Paris

Marcel JOUSSE

Professeur d'Anthropologie linguistique

à l'École d'Anthropologie

Directeur du Laboratoire de Rythmo-pédagogie de Paris

\title{
Du Mimisme à la Musique
}

\author{
chez l'Enfant
}

\section{PARIS}

\section{LIBRAIRIE ORIENTALISTE PAUL GEUTHNER}

12, Rue Vavin $\left(\mathrm{VI}^{\circ}\right)$

1935 


\section{- I - \\ Le Mimisme corporel et manuel}

$\underline{\text { Retour à la table des matières }}$

L'Enfant reçoit par les gestes de tout son corps, instinctivement mimeur, les Actions caractéristiques et les Actions transitoires des êtres animés et inanimés du Monde extérieur. En face du Mimodrame perpétuel de l'Univers, le "composé humain", fait de chair et d'esprit, se comporte comme un étrange miroir sculptural, infiniment fluide et sans cesse remodelé.

L'Enfant enregistre gestuellement ce Mimodrame universel aux cent actes divers, à la manière d'un film plastique, vivant et fixateur. Il devient, sans le savoir, un complexus de Mimèmes ou gestes mimismologiques intussusceptionnés. Leur richesse s'accroît à chaque intussusception nouvelle.

L'Enfant rejoue mimismologiquement par les gestes de tout son corps, et surtout par les gestes de ses mains innombrables, les phases de chaque Interaction de l'Univers. Ce qui s'est fait physiquement et inconsciemment dans l'Univers se refait psycho-physiologiquement et consciemment dans l'Enfant.

Ce rejeu des Mimèmes corporels et manuels n'est pas éparpillé ni incohérent. Il s'exécute spontanément sous la forme intelligente et logique d'un geste propositionnel, généralement triphasé: 


\section{L'Agent agissant sur l'Agi.}

Ces trois phases normales du geste propositionnel mimismologique sont nécessairement successives, mais elles sont aussi biologiquement imbriquées. Elles forment un "tout" musculaire et sémantique indéchirable.

Dès lors, la Pensée vivante de l'Enfant a son vivant outil de conquête, de conservation et d'expression du Réel: le Mimage ou Langage par gestes corporels et manuels, mimismologiques et propositionnels.

C'est sur cette base intellectuelle et vivante du rejeu propositionnel mimismologique que devra être fondée toute la Pédagogie anthropologique.

La Pédagogie sera désormais une Mimo-pédagogie. 


\section{- II - \\ Le Parallélisme propositionnel}

$\underline{\text { Retour à la table des matières }}$

Par suite de la conformation bilatérale du corps humain, les gestes propositionnels du Style corporel et manuel ont une tendance à rejouer en se balançant rythmiquement deux par deux, plus rarement trois par trois.

C'est la grande loi anthropologique du Parallélisme des gestes propositionnels. Nous en retrouverons l'influence ou la survivance partout, et spécialement dans les activités intellectuelles suivantes:

$1^{\circ}$ Dans les balancements alternés de ce que nous appelons "Danses populaires", ces cadavres de pensée, résidus purement gymnastiques et désormais méconnaissables des antiques Rythmo-mimiques propositionnelles et pédagogiques. Ces balancements corporels sont si profondément physiologiques que, seuls, ils ont pu survivre à la dégradation millénaire et progressive des Rythmo-mimiques propositionnelles. Cependant, c'était l'élément propositionnel qui constituait la grandeur souveraine de ces gestes humains. L'Animal a des Danses gymnastiques. Seul, l'Homme a des Rythmo-mimiques propositionnelles. Seul, il a le mystérieux privilège de "propositionner" ses gestes. La Proposition est le miracle de la Vie.

$2^{\circ}$ Dans les hémistiches isosyllabiques et balancés de ce que nous appelons "Chansons populaires", résidus banalisés des antiques Récitatifs du Style oral 
rythmo-pédagogique. On connaît les avatars du Style oral dans nos milieux ethniques de Style écrit. A un moment donné, des écrivains, parfois génies de premier ordre, imitèrent graphiquement et servilement les formes traditionnelles et balancées du Style oral. Naturellement, ils ne comprenaient plus la nature psycho-physiologique et mnémonique de ces balancements monotones, mnémotechniquement enchaînés par des rimes. Ils n'y cherchaient qu'un plaisir esthétique. Aussi, à la longue, s'en lassèrent-ils. De là, au siècle dernier, la révolte vraiment bien tardive de ces scribes contre la monotonie du traditionnel balancement des hémistiches isosyllabiquement parallèles. De là, peu après, l'avènement logique du vers libre, sans rimes, si parfaitement "ennemi de la mémoire". Ainsi abandonné et méprisé depuis des siècles par l'élite intellectuelle, le style oral mnémonique et mnémotechnique de nos Druides et de nos Trouvères s'est réfugié dans nos Chansons populaires où il attend sa réhabilitation pédagogique.

$3^{\circ}$ Dans les “membres" balancés des traditionnelles mélodies qui animent toujours rythmo-pédagogiquement les balancements propositionnels oraux de ces Chansons populaires, transmises de mémoire. Vidés de leurs paroles, les vivants balancements mélodiques sont devenus notre Musique instrumentale, de plus en plus algébrisée. 


\section{- III - \\ Du Mimisme au Mimographisme}

$\underline{\text { Retour à la table des matières }}$

Les muscles corporels et manuels de l'Enfant sont débordants de Mimèmes que l'intussusception mimismologique des Actions de l'Univers a modelés en lui. L'Enfant "joue" à tout, répétons-nous couramment. Ce n'est pas exact : il "est joué" par tout. Ces Cinémimèmes lui sortent, pour ainsi dire, par tous les gestes.

Ils lui sortent, d'une façon invisible pour nous, par les gestes oculaires. C'est ce rejeu spontané des Mimèmes oculaires que nous appelons "le rêve chez l'Enfant".

Ils lui sortent, d'une façon visible pour nous, par tous les gestes corporels et manuels. c'est ce rejeu spontané des Mimèmes corporels et manuels que nous appelons "le jeu instinctif chez l'Enfant".

N'oublions pas pourtant que, dans le rêve et dans le jeu instinctif, malgré la trompeuse différence de notre appellation, le mécanisme psycho-physiologique est de même nature. C'est un rejeu gestuel de Mimèmes préalablement intussusceptionnés.

Ces deux claviers de rejeu sont d'ailleurs fonctionnellement interdépendants. Les microscopiques Mimèmes oculaires irradient et s'amplifient dans les macroscopiques Mimèmes corporels et manuels. C'est cette amplifiante irradiation gestuelle que nous 
exprimons chaque jour en disant: "L'Enfant joue son rêve". Nous pourrions tout aussi bien dire: "L'Enfant rêve son jeu". En réalité, c'est l'incoercible loi anthropologique du Mimisme oculaire et corporel qui fonctionne en contraignant l'Enfant à prendre, à apprendre et à comprendre, par le rejeu propositionnel, les Interactions de l'Univers. Le jeu est la science de l'Enfant.

Débordant ainsi gestuellement de Mimèmes, l'Enfant ne peut s'empêcher de les projeter mimismologiquement sur les murs, sous la forme gesticulante d'"ombres chinoises" qu'il fait se combattre les unes les autres. Bien mieux, dès qu'il a en main un charbon ou un crayon, il "réifie" ces évanescents Mimèmes propositionnels sous la forme de Mimogrammes ou Dessins spontanés. Ainsi les premiers Hommes, au stade du Mimage, avaient-ils commencé à écrire par Mimogrammes pictographiques et propositionnels.

De même que l'Enfant est un Mimeur né, il est un Dessinateur né. Loin d'inhiber le Mimographisme instinctif, en condamnant prématurément l'Enfant à notre écriture algébrisée et rebutante pour lui, la Mimo-pédagogie s'ingénie à en tirer le maximum de rendement intellectuel et scientifique. Le dessin est l'écriture de l'Enfant. 


\section{- IV - \\ Le Phonomimisme auriculaire}

$\underline{\text { Retour à la table des matières }}$

Les êtres animés et inanimés de l'Univers n'ont pas seulement des Actions plastiques et visibles. Ils ont aussi des Actions sonores et audibles qui se répercutent mimismologiquement dans les gestes microscopiques de l'oreille interne sous la forme de Mimèmes auriculaires. C'est le Phonomimisme auriculaire.

Jusqu'en ces dernières années, dans nos milieux ethniques trop livresques et trop artificiels, l'oreille de l'Enfant n'a presque jamais été pédagogiquement initiée aux subtiles délicatesses de l'audition des choses. Ses gestes auriculaires ne sont guère modelés que par les quelques sons stéréotypés de nos langues gréco-latines algébrisées et par les quelques notes mécaniques de notre musique instrumentale.

Or, ce langage et cette musique appauvrissent très vite les riches potentialités de l'oreille enfantine. Les appareils enregistreurs de la Phonétique expérimentale nous ont révélé que l'oreille d'un adulte ne peut plus "entendre" objectivement les phonèmes d'un idiome inconnu. Elle les déforme subjectivement en les réduisant à la plus simple expression des phonèmes propres aux langues apprises dès l'enfance. Les spécialistes européens des mélodies orientales nous ont dit également leur impuissance à "saisir" ces mélodies dans leurs particularités sonores. La gesticulation auriculaire, originellement si fluide, se présente alors comme sclérosée en un nombre restreint de gestes réceptifs, désormais invariables. 
Notre langage et notre musique concentrent donc trop exclusivement l'oreille enfantine sur les Algébrèmes sonores des Signes au lieu de la laisser s'assouplir par les Mimèmes sonores des choses. Utilitaires et artistes à courte vue, nous avons hâte d'apprendre à l'Enfant le nom social des choses et les notes en série de nos gammes. Par malheur, nous négligeons de lui faire entendre aussi le timbre caractéristique des choses elles-mêmes. Le maniement si facile du mot socialisé et de la note algébrisée tue bientôt la curiosité spontanée pour le son concret du vrai Réel.

Cependant, au point de vue intellectuel comme au point de vue esthétique, l'harmonie inattendue des sons de la nature n'est pas moins formatrice que l'harmonie stylisée des notes d'un orchestre. L'oreille avide d'Eschyle n'a-t-elle pas rendu inoubliable "l'innombrable éclat de rire des flots marins ?" Les sonorités qu'une oreille humaine a déjà entendues peuvent être douces, mais combien plus douces celles qu' aucune oreille n'avait jamais encore su entendre ! Le Réel harmonieux est plus riche que notre dictionnaire et plus nuancé que notre musique. 


\section{$-\mathrm{V}-$ \\ Le Phonomimisme oral}

$\underline{\text { Retour à la table des matières }}$

Comme le Cinémimisme oculaire, le Phonomimisme auriculaire se joue secrètement en gestes microscopiques et sur des organes jusqu'ici inaccessibles à l'observation d'autrui. Le maître ne voit pas les rejeux oculaires de l'enfant, il n'entend pas ses rejeux auriculaires. Aucun contrôle pédagogique n'est donc directement possible.

Par bonheur, de même que le Cinémimisme oculaire s'amplifie dans le Cinémimisme corporel et manuel, le Phonomimisme auriculaire a, lui aussi et spontanément, son amplifiante irradiation en écho sur la musculature laryngo-buccale. Le son qui s'est joué mimismologiquement et microphoniquement dans l'oreille interne a tendance à se rejouer mimismologiquement et mégaphoniquement sur les lèvres. C'est le Phonomimisme oral.

C'est en vertu de cette nouvelle spécialisation de la loi générale du Mimisme qu'une jeune enfant, élevée uniquement par sa mère, se trouve inconsciemment proférer les timbres et les inflexions caractéristiques de la prononciation maternelle. On entend la voix de la mère dans la voix de sa fille, comme on voit les gestes de la mère dans les gestes de sa fille. Nous sommes ici aux sources vives et profondes de ce qu'on a si judicieusement appelé : la “contagion” de l'exemple.

C'est naturellement sous cette propulsante contrainte du Phonomimisme auriculaire et oral que tout enfant, élevé en liberté à la campagne, se met de lui-même à nommer un certain nombre d'animaux et d'objets par le son caractéristique qu'ils 
émettent. Témoin ce jeune enfant sarthois, parlant à peine, et qui accourait vers sa mère la fermière, en portant rythmiquement la main à la bouche et en criant avec des intonations mélodiques d'une saisissante justesse:

\section{Miaou ham cô ! Miaou ham cô !}

Un gros et redoutable chat du voisinage venait effectivement d'étrangler une poulette.

Si tel ou tel enfant est doué d'un phonomimisme auriculaire très précis, il intussusceptionne et rejoue auriculairement, avec une plus grande justesse, le son caractéristique émis par chaque chose, avec son intensité, sa durée, sa hauteur et son timbre. Il a donc, d'ordinaire, un phonomimisme oral plus exact. Mais ce phonomimisme oral en écho peut avoir ses déficiences propres. L'oreille peut être juste et l'appareil laryngo-buccal faux. Ce dernier peut même, par mimisme en retour, arriver à fausser les phonomimèmes auriculaires.

La Mimo-pédagogie doit donc intervenir, dès les premières années, pour vérifier et ajuster ces deux systèmes phono-mimismologiques. 


\section{- VI - \\ Du Mimage au Langage}

$\underline{\text { Retour à la table des matières }}$

Sans se soucier du Mimisme humain, notre petit enfant sarthois n'était pourtant plus, lui aussi, qu' un "écho sonore" et plastique d'une Action plastique et sonore de l'Agent agissant sur l'Agi. Ce Mimodrame se rejouait, s'intelligeait et s'exprimait en lui dans les trois phases imbriquées d'un geste propositionnel, manuellement et oralement mimismologique et bilatéralement balancé.

Pour les gestes mimismologiques corporels et manuels, modelés en lui par l'intussusception des Actions plastiques des choses, nous avons vu que l'Enfant "est joué” par elles beaucoup plus qu'il n'y “joue”. Nous constatons maintenant la même impulsion spontanée au sujet des gestes mimismologiques oraux, dès que l'Enfant est mis en contact direct et vivant avec les Actions sonores des choses. Or, le jeu oral est aussi impoortant que le jeu corporel pour la formation et l'enrichissement de la pensée enfantine. L'observation de l'Enfant, laissé à ses activités instinctives, nous en donne des preuves quotidiennes. Il est aussi spontanément curieux d'écouter et de rejouer les timbres caractéristiques des choses qu'il est spontanément curieux de regarder et de rejouer leurs formes et leurs actions caractéristiques.

C'est d'ailleurs cette curiosité spontanée qui a jadis permis à l'Homme corporellement mimeur de devenir phonétiquement, lingualement mimeur. Le Mimage (ou expression intellectuelle par les gestes plastiques du corps et des mains) a cédé ainsi 
peu à peu, mais jamais complètement, son admirable puissance significative au Langage (ou expression intellectuelle par les gestes sonores de la langue). Les premiers langages oraux ont été dictés aux divers groupements ethniques d'hommes de Style manuel par la voix même des choses, avec les vivantes variantes dues aux rejeux naturellement variables des vivants et intelligents organes récepteurs: Mimisme humain n'est pas Machinisme brutal. Le petit enfant sarthois, dans toute sa fraîcheur d'"Anthropos éternel", nous a expérimentalement montré l'authenticité de cette dictée aux oreilles humaines spontanément attentives.

L'examen des langues, moins algébrisées que les nôtres, nous la prouve aussi nettement par la quantité de phonomimèmes ou "onomatopées" qui ont résisté à la millénaire dégradation articulatoire des évolutions phonétiques. Les Chinois et les Annamites, entre beaucoup d'autres, nous détaillent avec un légitime orgueil toute la richesse et la subtile finesse des innombrables onomatopées qui sont toujours vivantes sur leurs lèvres et toujours senties dans leurs oreilles. Ainsi sont encore aperçus et admirés, sous le tracé algébrisant du pinceau, les concrets mimogrammes ou "ombres chinoises" de leur Style manuel de jadis. 
Du Mimisme à la Musique chez l'enfant (1935)

\section{- VII - \\ Du Langage au Style oral}

$\underline{\text { Retour à la table des matières }}$

Les gestes propositionnels corporels et manuels, propulsés bilatéralement par les déflagrations successives de l'énergie vivante, se balancent par Binaires, et plus rarement par Ternaires, selon un rythme spontané. Dans la matière vivante, le Rythme est le retour d'un même phénomène physiologique à des intervalles biologiquement équivalents. Régularisé, il devient le Mètre.

Chaque balancement propositionnel des Binaires et des Ternaires est généralement triphasé, puisque se rejouent en lui les Mimèmes de l'Agent, de l'Interaction et de l'Agi. A l'intérieur de chaque balancement, ces trois éléments gestuels sont individuellement propulsés et scandés, eux aussi, par les intensifications et détentes successives de l'énergie qui s'épanouit dans la durée. Nous nous trouvons donc, dès maintenant, en présence des deux rythmes fondamentaux, inhérents à toute série de gestes vivants : le rythme d'intensité et le rythme de durée.

Lorsqu'ils sont transposés sur les muscles laryngo-buccaux, les gestes propositionnels restent balancés. Ils conservent aussi, forcément, leurs deux rythmes fondamentaux d'intensité et de durée. Mais les gestes laryngo-buccaux deviennent émetteurs de sons qui peuvent varier par leur hauteur et par leur timbre. Dans chacun des balancements des Binaires et des Ternaires oraux, nous aurons donc quatre rythmes : le rythme d'intensité, le rythme de durée, le rythme de hauteur et le rythme 
de timbre. Selon les évolutions phonétiques propres à chaque langue, l'un ou l'autre de ces quatre rythmes omniprésents pourra devenir spontanément prédominant dans une langue donnée. Il tendra alors à imposer ses schémas régulateurs à toutes les propositions de cette langue. Étant plus automatique, il facilitera grandement l'improvisation, la mémorisation et la remémoration. Peu à peu, se développera le mécanisme traditionnel du Style oral rythmo-pédagogique qui modèlera sur ses propres structures rythmiques les structures rythmiques de ses mélodies. C'est du tréfonds même d'une langue que jaillit originellement la mélodie.

Mais une langue est une chose vivante et changeante. La mélodie, sans être immuable, a plus de stabilité rythmique. Des mélodies, séculairement préformées, imposeront donc parfois leur rythme archaïque et fausseront ainsi le rythme nouveau des propositions qu'elles devraient au contraire renforcer. Par exemple, analysons phonétiquement nos Chansons populaires, précieux résidus de notre ancien Style oral. nous y constaterons que le rythme d'intensité de la mélodie ne coïncide presque jamais avec le rythme d'intensité des paroles. L'évolution phonétique de notre langue en est cause. 


\section{- VIII - \\ Du Style oral à la Musique}

$\underline{\text { Retour à la table des matières }}$

Le Style oral du latin populaire, tel qu'il fut apporté en Gaule, avait ses balancements verbaux rythmés par ïambes ou trochées d'intensité. La mélodie, infuse en ce Style oral, le scandait normalement selon ce rythme. Mais très vite dans notre bouche, semble-t-il, l'explosion énergétique de l'articulation s'est faite de moins en moins intense. Sauf à la fin de chaque balancement, n'importe quelle syllabe, même faible, pouvait venir s'intensifier sous les explosions énergétiques intérieures, toujours très nettes, de la mélodie traditionnelle. Il en est encore ainsi. À peu près aucun français, même poète ou musicien, ne sent ni ne sait le rythme des phrases qu'il articule. Il a fallu les récents enregistrements de la Phonétique expérimentale pour nous prouver que le rythme dominant du français actuel est encore le rythme d'intensité, tendant vers l'anapeste. Mais cette intensité est toujours si douce et si étalée qu'elle permet, même à des phonéticiens nés à Paris, de ne sentir, comme prédominant en français, que le rythme de durée.

Mimeur né, Dessinateur né, l'Enfant est aussi, comme notre petit Sarthois, un Rythmeur-Improvisateur né, soit qu'il rejoue le son des choses en mélodiques onomatopées, inlassablement parallèles, soit qu'il commente en son Style oral français à lui, toujours si rythmique et si mélodique, les gestes balancés de ses Rythmomimiques. Captons ces rejeux rythmo-mélodiques verbaux pour les prolonger insensiblement et pédagogiquement par les courtes phrases rythmo-mélodiques de notre 
Style oral, tel qu'il se survit dans nos Chansons populaires. Mais rectifions avec soin les paroles de ces Chansons pour que les deux rythmes d'intensité, le verbal et le mélodique, coïncident. L'intime rythmisation de l'Enfant par le rythme exact et mélodique de son langage, irradié par son geste rythmo-mimismologique corporel et manuel, devrait être le premier souci des éducateurs. Les Grecs harmonieux l'avaient compris. Ils faisaient mélodiquement rythmo-mimer à leurs enfants le Style oral dactylique d'Homère. Nous aussi, basons sur le rythme du Langage toute initiation à la Musique qui, historiquement, en est jaillie. Le vrai problème n'est pas d'apprendre prématurément à l'Enfant comment il va lire, écrire et jouer des sons vides. L'être humain jeune, pas plus que l'humanité jeune, ne saurait, sans transitions lentes, dissocier la Musique pure d'avec la Parole qui est anthropologiquement geste rythmomimismologique significatif en même temps que mélodie.

Une fois maître de cette complexe et vivante lyre qu'est son propre corps, l'enfant maîtrisera, comme en se jouant, les techniques les plus algébrisées de nos instruments de musique morts. Sa main musicale rendra musicales toutes choses.

Fin du mémoire. 